Aus der Universitäts-Klinik für Ohren-, Nasen- und Halskrankheiten in Kiel. (Direktor: Prof. Dr. Zimmermann.)

\title{
Zur Frage der Schädigungen des Nervus VIII bei frischer Lues.
}

Von Dr. Walther Brünìng, Volontärassìstent der Klinik.

In ,P a ssow s Beiträge" I920, Bd. I5, H.x -2 veröffentlichte Kobrak eine Arbeit: „Untersuchungen des Nervus Octavus bei rezenter Lues; Erregbarkeit vor und am Ende einer kombinierten QuecksilberSalvarsankur."

Kobrak stellte seine Untersuchungen an, um die Frage zu klären, ob die Neurorezidive des Akustikus, die mit Ejnführung des Salvarsans in die antiluetische Therapie so häufig und so frühzeitig auftraten, durch direkte Schädigungen des Nerven bei der spezifischen Behandlung mit Quecksilber und Salvarsan entstünden oder nicht.

$\mathrm{Zu}$ diesem $Z$ weck untersuchte er Io6 Fälle rezenter Lues (d. h: Fälle des I. Stadiums und der Frühsekundärperiode; auch einige Rezidivé älterer Lues rechnete er dazu) vor Beginn irgendeiner antiluetischen Kur und dann wieder am Ende der Kur. Kobrak nahm diese Reihenuntersuchungen vor bei luetisch infizierten Patienten ohne Rücksicht darauf, ob irgendwelche subjektiven Symptome einer luetischen Erkrankung des Nervus VIII vorhanden waren oder nicht, in der Erkenntnis, daß man erst dann $\mathrm{zu}$ einem richtigen Ergebnis kommen könne, wenn man die Patienten möglichst bald nach der luetischen Infektion beobachte und auf Akustikusstörungen untersuche, ohne erst subjektive Beschwerden abzuwarten. Gerade die luetische Affektion des Oktavus läßt ja im Anfangsstadium die Patienten subjektiv vielfach vollkommen beschwerdefrei, und nur einer genauen Untersuchung bleibt es in diesen Fällen vorbehalten, die trotzdem bereits erfolgte Erkrankung festzustellen. Erst wenn man vor Beginn und am Ende der Kur den Nervus cochlearis und vestrbularis untersucht, läßt sich erkennen, ob ein Unterschied zwischen Anfangs- und Schlußbefunden besteht, und damit feststellen, welchen Einfluß ev. die spezifische Behandlung auf den gesunden oder geschädigten Nerven ausgeübt hat. 
Die Kobrakschen Untersuchungen ergaben, da 3 von 106 vor der Kur untersuchten Fällen nur io völlig oktavusgesund waren, daß dagegen $87-82 \%$ eine Störung des Akustikus aufwiesen und zwar:

$6 x-58, x \%$ schwache Cochlearisaffektionen

$8-7,4 \%$ deutliche

$33-3 I, I \%$ schwache Vestibularisaffektionen

$I 5-I 4, I \%$ deutliche

Davon waren beide Nerven in I F Fallen leicht, in einem Fall schwer ( $18-\mathrm{I} 6,9 \%$ ) gleichzeitig erkrankt.

In 8 Fällen $=7,5 \%$ wurden Erkrankungen des Nervus VIII gefunden bei noch negativem Wassermann.

Am Ende der Kur wurden festgestellt:

am N.-Cochlearis: am N.-Vestibularis:

Deutliche Verschlechterungen $\quad 7 \mathrm{mal} \quad 9 \mathrm{mal}$

Schwache " 5 ", 9 ,"

Deutliche Besserungen 7 ". 6 ,

Schwache " 6 ,, I5 ,

Kobrak stellte somit einen außerordentlich hohen Prozentsatz von Erkrankungen des Akustikus bei frischer Lues schon vor Beginn jeder spezifischen Behandlung fest.

Auf Grund seiner Anfangs- und Schlußbefunde glaubte Kobrak, eine direkte Schädigung des Nerven durch Quecksilber und Salvarsan ablehnen zu dürfen. A. Alexander ( $\boldsymbol{I}$ ), der mit Kobrak zusammen arbeitete, wies allerdings ausdrücklich darauf hin, daB diese Frage mit voller Sicherheit nur entschieden werden könne, wenn man systematische Reihenuntersuchungen von Patienten mit ausschließlich seronegativen Primäraffekten vornehme und zwar exakte Oktavusprüfungen vor und am Ende der spezifischen Kur.

Diese Ergebnisse der Kobrakschen Untersuchungen, die zu der ziffernmäßigen Feststellung führten, daß von Io6 luetisch infizierten Patienten vor Beginn irgendeiner antiluetischen Behandlung nur Ig keine Erkrankung des Akustikus aufwiesen, schienen uns nach den hier gemachten Beobachtungen für unser Luesmaterial nicht zuzutreffen.

Zur Klärung dieser Divergenz' wurden im Sommer I920 genaue fortlaufende Untersuchungen der uns von der hiesigen Hautklinik (Direktor Prof. Dr. Klingmüller) nach vorausgegangener Vereinbarung systematisch zugeschickten Patienten mit frischer unbehandelter Lues, und zwar Fälle des I. Stadiums und der Frühsekundärperiode aufgenommen. Mit diesen Untersuchungen war zunächst der damalige I. Assistent der Klink Dr. Meye betraut, bei dessen Abgang von der Klinik übernahm ich dann die Forführung der Arbeit. 
Wir waren uns daräber klar, daß unser Ergebnis nur dann einigermaßen den tatsächlichen Verhältnissen entsprechen konnte, wenn alle bei derartigen statistischen Erhebungen erfahrungsgemäß leicht unterlaufenden Fehlerquellen so gut wie nur irgend möglich vermieden würden. Eine der in dieser Hinsicht wichtigsten Voraussetzungen war die sorgfältige Auswahl des Materials. In unsere Serie wurden infolgedessen nur Fälle aufgenommen, bei denen das Vorliegen einer Hörschädigung durch frühere Mittelohrerkrankungen und deren Komplikationen, Traumen, Kriegsverletzungen, Berufsstörungen, Altersschwerhörigkeit usw. nach Möglichkeit ausgeschlossen werden konnte.

Bezüglich der Untersuchungsmethoden folgten wir Kobrak, um nicht durch eine Abweichung die Gleichmäßigkeit der Methodik und dadurch die Vergleichbarkeit der Resultate in Frage zu stellen. Nur konnten wir uns nicht entschließen, den Vestibularis durch „Digitalkompression" zu prüfen, einmal, weil wir in der Wertung der bei dieser Prüfungsmethode sich ergebenden Befunde keine genügende Erfahrung besaßen, und dann, weil Kobrak selbst nur die Hälfte seiner Fälle so untersucht hatte.

Wir beließen es daher in allen Fällen bei der kalorischen Reizung.

Wir untersuchten also demgemäß:

den Nervus Cochlearis durch Prüfung mit Flüstersprache und der kontinuierlichen Tonreihe, den Nervus Vestibularis durch:

I. Nachfrage nach subjektivem Schwindelgefühl,

II. Prüfung auf objektiv nachweisbare Gleichgewichtsstörungen,

III. auf Spontannystagmus,

IV. sowie durch die kalorische Prüfung nach Bárány.

Auf diese Weise haben wir 5 Fälle Lues I und 5o Fälle der Frühsekundärperiode untersucht. Das Ergebnis war das folgende:

I. Bei den 5 Fällen Lues $I=9 \%$ aller Fälle (die. WassermannReaktion war noch negativ!) fand sich bei allen Patienten normale Hörfunktion und regelrechte Erregbarkeit des Vestibularis.

II. Bei den 50 Fällen der Frühselundärperiode ergab sich,(die Wassermannsche Reaktion war in allen Fällen positiv):

a) Bei 37 Fällen $=67,3 \%$ vollkommene Unversehrtheit beider Teile des Nervus VIII.

b) Bei 3 Fällen $=5,5 \%$ war der Cochlearis erkrankt, d. h. es fand sich eine Herabsetzung der oberen Tongrenze, während Flüstersprache normal weit gehört wurde. (In èinem Fall war gleichzeitig die Kopfknochenleitung stark verkürzt.) 
c) Bei Io Fällen $=18,2 \%$ war der Vestibularis erkrankt:

8 mal übererregbar,

2 mal untererregbar,

in allen Io Fällen war der Cochlearis unbeteiligt.

Bei einem Teil der Patienten mit L. II war die Lumbalflüssigkeit untersucht worden und zwar:

bei den 37 ohrgesunden 23 mal mit 3 mal schwach positivem Befunde, bei den 3 Cochleariskranken I mal mit positivem Resultat,

bei den Io Vestibulariskranken $6 \mathrm{mal}$, 3 mal mit positivem, $3 \mathrm{mal}$ mit negativem Ergebnis.

Von den 55 Patienten waren 2 I männlich, 34 weiblich.

Es erkrankten am Cochlearis 3 weibliche, am Vestibularis 6 weibliche, 4 männliche Patienten.

Alle 55 Patienten gaben an, gut zu hören und bis dahin niemals an Ohrensausen oder Schwindel gelitten zu haben.

Im Gegensatz zu Kobrak haben demnach Paralleluntersuchungen für das Material unserer Klinik ergeben:

I. Der Prozentsatz der Erkrankungen des Nervus VIII bei frischer Lues ist lange nicht so hoch, wie von Kobrak an seinem Krankenmaterial festgestellt.

$23,6 \%$ bei unseren Fällen, gegen $82 \%$ in Kobraks Serie.

II. Der N.-Vestibularis erkrankt häufiger isoliert wie der N.-Cochlearis, bei uns ro:3, bei. Kobrak 6:9 (für den Vergleich zwischen isolierten V.- und C.-Erkrankungen zog K. nur die 42. Fälle heran, in denen der Vestibularis wie bei uns nach Bárảny geprüft wurde, da nach seiner Ansicht bei den Prüfungen lediglich durch Digitalkompression etwaige Untererregbarkeitszustände übersehen werden könnten).

Bei diesen 42 Fällen fand er 8 leichte und eine schwere Cochlearisaffektion, sowie 6 leichte Vestibularisschädigungen.

III. Eine Erkrankung beider Nerven ist in keinem Falle bemerkt worden, bei Kobrak in $16,9 \%$.

IV. Bei frischer Lues mit noch nicht positivem Wassermann ist eineAffektion des Nervus VIII nicht festgestellt, bei Kobrak in $7 \%$ der Fälle der Vorwassermannperiode.

Betrachten wir das Ergebnis unserer Untersuchungen kritisch. Ob die von uns nach erfolgter Infektion, aber vor Einleitung der spezifischen Behandlung festgestellten 23,6\% Akustikusaffektionen nun wirklich alle luetischer Natur sind, ist eine Frage, die wir nicht mit Sicherheit zu klären vermögen.

Es ist keineswegs unmöglich, daß sich auch in unserer Serie, trotz sorgfältigster Auswahl des Einzelfalles, doch manche Oktavusschädi- 
gung nicht-luetischer Provenienz (z. B. infolge beginnender Otosklerose, auf Grund eines früher erlittenen, längst in Vergessenheit geratenen Traumas, eines alten toxischen oder infektiösen Labyrinthbzw. Akustikusprozesses usw.) befindet, und daß daher der Prozentsatz der tatsächlich luetischen Hörstörungen noch geringer ist, als es sich in dem gefundenen Prozentverhältnis 23,6: 100 ausdrückt.

Das Uberwiegen der weiblichen Kranken in unserer Statistik ist wohl dadurch zu erklären, daß bei den männlichen Patienten viele, die Kriegsteilnehmer waren, wegen des Verdachtes auf eine Kriegsstörung nicht in die Untersuchungsreihe aufgenommen wurden.

$\mathrm{Da}$ wir weniger Cochlearisaffektionen fanden als Kobrak, scheint uns durch die schon eingangs erwähnte Art der Auswahl unserer Fälle begründet zu sein. Schieden wir doch von vornherein alle Fälle aus, die früher irgendeine dem Patienten bekannte Hörschädigung, wenn auch nur der leichtesten Art, erlitten hatten. Selbst Fälle, bei denen schon theoretisch Berufs- und Altersstörungen u. a. m. hätten in Frage kommen können, nahmen wir in unsere Statistik nicht auf:

Dies hat Kobrak, soweit aus seiner Publikation zu ersehen ist, nicht getan. Daher finden sich, worauf übrigens Kobrak (wenigstens für die männlichen Patienten) in seiner Arbeit selbst hinweist, unter seinen oktavuskranken Fällen, und zwar nicht nur unter seinen männlichen Patienten, höchst wahrscheinlich auch solche, bei denen die von ihm festgestellte und als luetischi angenommene Ohraffektion nicht luetisch war.

Einen Teil seiner Fälle von Cochleariserkrankungen (4I) bezeichnet Kobrak selbst ferner deshalb für seine Statistik nicht als einwandfrei, weil sie sich nur durch Veränderungen für Flüstersprache oder durch eine leichte Einschränkung für Stimmgabeltöne geltend machten, und K. somit selbst die Möglichkeit von: Beobachtungsfehlern konzediert. Wir halten es infolgedessen nicht für angängig, bei allen derartigen Fällen der luetischen Affektion die Schuld zu geben. Eine geringe Herabsetzung der oberen Tongrenze bei normaler Hörfähigkeit für Flüstersprache und umgekehrt eine geringe Herabsetzung der Hörfähigkeit für Flüstersprache bei normalem Stimmgabelbefund findet man doch in mancherlei Fällen, die niemals etwas mit Lues zu tun hatten.

U. E. sind als wahrscheinlich luetisch Schädigungen der Hörfunktion nur dann zu betrachten und in eine derartige Statistik aufzunehmen, wenn man bei einem luetisch infizierten Patienten unter möglichst scharfem AusschluB jeder anderweitigen, für die Entstehung einer Labyrinthaffektion in Frage kommenden Schädigung die für Cochleariserkrankung deutlichen Symptome (vor allen Dingen deutliche Herabsetzung der oberen ev: Heraufrücken der unteren Tongrenze, in vielen Fällen auch Verkürzung der Kopfknochenleitung und Verminderung der Hörfähigkeit für Flïstersprache) gefunden hat. 
Die Feststellung einer verminderten Hörfähigkeit für Flüstersprache allein, ein Kriterium, wie es Kobrak in einer Reihe seiner Fälle verwandt hat, kann unseres Erachtens nicht genügen.

So sei hier nur darauf hingewiesen, daß $O$. Beck, Wanner, Gustav Alexander ( 2 ) u. a. der Tatsache, daß man in einem Teil der Fälle eine relativ bedeutende Verkürzung der Kopfknochenleitung bei sonst normalem oder annähernd normalem Hörvermögen findet, eine große diagnostische Bedeutung zumessen. O. Beck fand dieses Symptom bei $80 \%$ der normalhörenden Luetiker. Ja, nach Alexanders Ansicht kann man sogar aus dem Mißverhältnis zwischen Verkürzung der Kopfknochenleitung und Hörvermögen in ätiologisch unklaren Fällen mitunter die Lues diagnostizieren.

Wir fanden jedoch nur in einem Falle bei sonst vollkommen normaler Hörfunktion eine beträchtliche Verkürzung der Kopflknochenleitung, also, ähnlich wie Kobrak, relativ selten. Vielleicht hängt das damit zusammen, daß, wie Kobrak anzunehmen geneigt ist, sich sein und unser Material aus meist symptomlosen, frischen, unbehandelten Fällen zusammensetzt.

Auch bei den Erkrankungen des Vestibularis bleiben wir hinterKobraks Zahl zurück.

Kobrak hat bei seinen Vestibularisuntersuchungen zwei Methoden angewandt, die Báránysche und die Digitalkompression, und zwar hat er $6_{4}$ Fälle durch Digitalkompression geprüft und 42 nach Bárány. Darunter leidet zweifellośs die Beurteilbarkeit des Untersuchungsergebnisses. Kobrak teilt darüber mit: „Es scheint, als sei die Kompressionsmethode für Übererregbarkeitszustände der feinere Indikator, so daß bei der Spülungsmethode Übererregbarkeitszustände übersehen werden könnten, die durch die Kompressionsmethode aufgedeckt würden".

Es ist eine Frage, ob bei gleichmäßiger Durchführung einer Prüfungsart nicht ein wesentlich anderes Ergebnis erzielt worden wäre, Voraussetzung ist auch hier die Ausscheidung aller Fälle, bei denen die Möglichkeit einer früher erlittenen Labyrinthschädigung in Frage kommen kann.

Weshalb der Vestibularis häufiger luetisch erkrankt wie der Cochlearis, darüber haben uns unsere Untersuchungen keinen Aufschluß gegeben. Vielleicht ist der Vestibularis doch empfindlicher wie derCochlearis.

Mit dieser Auffassung setzen wir uns allerdings in Gegensatz zu älteren Autoren, wie Benario (2) und Gustav Alexander.

Benario fand unter 44 Oktavusaffektionen:

Ig isolierte Cochlearisaffektionen,

I2 isolierte Vestibularisaffektionen,

I3 kombinierte C.- und V.-Erkrankungen. 
Gustav Alexander (2) betont ausdrücklich, daß die isolierten Vestibularisschädigungen außerordentlich selten seien, meist sei der Cochlearis (wenn auch nur in sehr geringem Grade) miterkrankt. Er meint, die theoretische Grundlage, da $B$ bei Lues uberhaupt isolierte Cochleariserkrankungen auftreten könnten, liege darin, daß der Vestibularis denselben Noxen gegenüber ungleich widerstandsfähiger sei, wie der Cochlearis.

Kobrak kommt unserer Ansicht schon näher, wenn er mitteilt, daß bei rezenter Lues isolierte Cochleariserkrankungen nicht nennenswert häufiger seien, wie isolierte Vestibularisaffektionen. Unser Resultat der häufigeren Erkrankung des Vestibularis ist eben sehr wahrscheinlich begründet durch unsere sorgfältige Auswahl der Fälle, durch die wir sicher manche Cochleariserkrankung nichtluetischer Herkunft ausgeschlossen haben. Jedenfalls ist durch die Kobrakschen, wie durch unsere eigenen Untersuchungsergebnisse einwandfrei festgestellt, daB es im Frühstadium dèr Lues eine isolierte Vestibularisaffektion gibt.

Was schließlich die Frage einer Oktavusschädigung bei negativem Wassermann betrifft, so stimmen auch hierin unsere Ergebnisse mit denen Kobraks nicht überein.

Kobrak fand unter seinen Io6 Fällen $8=7,5 \%$ Frühluetiker mit negativem Wassermann. Bei allen diesen 8 Fällen war eine Akustikusschädigung bereits nachweisbar.

Wir fanden bei 55 Fällen $5=9 \%$ Frühluetiker mit negativem Wassermann, bei keinem dieser 5 Fälle war jedoch der Oktavus nachweisbar erkrankt. Wodurch diese auffallende Differenz bedingt ist, können wir nicht entscheiden, vielleicht fällt eben auch hierbei der Auswahl der zur Statistik herangezogenen Einzelfälle eine gewisse die Resultate modifizierende Rolle zu.

Zweifellos ist ja, wie außer durch Kobrak, von Nathan (6) und Meirowski (5) einwandfrei festgestellt wurde, eine Erkrankung des Oktavus bei noch negativem Wassermann möglich. Die genannten Autoren haben Fälle beschrieben, bei denen der Wassermann von Anfang an negativ war, es auch während der weiteren exakten spezifischen Behandlung blieb und bei denen trotzdem Neurorezidive des Akustikus auftraten.

Daß aber solche Funktionsstörungen des Oktavus in der Vorwassermannperiode verallgemeinert so häufig auftreten, wie Kobrak angibt, halten wir nach unseren Befunden und nach den Mitteilungen der oben erwähnten Autoren nicht für wahrscheinlich, bzw. für nicht erwiesen. Allerdings ist das bis jetzt vorliegende Material noch zu klein, um ein endgültiges Urteil abgeben zu können. 
Auch die Frage eines ev. Zusammenhanges zwischen Liquorbefund und Akustikusstörung konnten wir nicht klären. Einmal, weil wir nur bei einem Teil unserer Fälle eine Lumbalpunktion vorgenommen hatten, und dann, weil wir sowohl bei akustikusnormalen. Patienten positive Liquorresultate, als auch umgekehrt bei Akustikusstörungen negative Liquorbefunde erhoben hatten. Jedenfalls fanden wir in mehreren Fällen mit stark positivem Liquorbefund deutliche Akustikusstörungen.

Unsere Arbeit war schon abgeschlossen, da erschien im ,Internationalen Zentralblatt für Ohrenheilkunde und Rhino-Laryngologie, Bd. I8, H. 9 u. Io" ein Bericht über die Habilitationsschrift Robert Lunds (4): „Neurolabyrinthitis syphilitica" (Kopenhagen I920).

Es war uns eine Genugtuung, feststellen zu können, daß sich die an einem achtfach größeren Material gewonnenen Lundschen Resultate entgegen den Kobrakschen Befunden mit unseren eigenen Ergebnissen nahezu decken.

Lund hat 5 I5 Fälle luetisch erkrankter Patienten untersucht, zur Statistik konnte er aber nur eine fortlaufende Reihe von 4.9 Fällen verwerten. (Davon waren 399 Patienten mit erworbener Lues.) Lund fand:

$27=6,6 \%$ isolierte C.-Erkrankungen, wir 5,5\%, Kobrak $48,6 \%$ ! $40=9,7 \% \quad$, V.- $\quad, \quad$,I $8,2 \% \quad$, $28,3 \%$ ! $I_{2}=4,8 \%$ kombinierte ", beider Nerven, wir $0 \%$, Kobrak I $6,9 \%$ !

Davon $3=0,73 \%$ Oktavusstörungen bei negativem Wassermann, wir in $0 \%$, Kobrak in $7,5 \%$.

Insgesamt 79 Fälle = etwa $20 \%$ (in unserer Statistik $23,6 \%$, bei Kobrak $82 \%$ !).

Von den 399 Patienten mit akquirierter Lues kamen 227 im ersten Jahre zur Untersuchung. Davon wiesen Ig\% eine luetische Akustikusstörung auf, während im zweiten Jahre II \% festgestellt wurden.

Nach Lunds Untersuchungen entsteher die meisten Oktavusschädigungen 2 Monate nach der Infektion und zwar meist gleichzeitg mit der ersten sekundären Eruption.

Bezüglich der Vestibularisaffektion betont Lund, daß die isolierten Vestibulariserkrankungen besonders häufig innerhalb des ersten Jahres aufträten. Er bestätigt somit das Vorkommen isolierter Vestibularisschädigungen im Frühstadium der Lues ebenfalls.

Auch er hat bèi einem großen Teil seiner Patienten (in fast 30\%) eine auffallende Verkürzung der Koptknochenleitung bei normalem Gehör für Flüstersprache festgestellt. 
Vergleichen wir die Ergebnisse der Lundschen Arbeit mit unseren Resultaten, so können wir sagen, daß unsere Zahlen (auch nach Abzug der wenigen Lund schen Fälle älterer Lues) annähernd übereinstimmen.

Auch Lund fand nur bei einem weit geringeren Teil seiner Patienten luetische Hörstörungen als Kobrals, nämlich in $20 \%$ gegen Kobrak in $82 \%$ und wir in $23,6 \%$.

Auch Lund fand mehr isolierte Vestibularerkrankungen wie Kobrak, wenn auch nicht in dem relativ hohen Prozentsatz wie wir.

Und ebenso stellte Lund fest, daß eine Affektion des Nervus VIII bei negativem Wasśermann nur äußerst selten vorkommt. Sein Ergebnis war $0,73 \%$ gegen Kobraks. $7 \%$ und unseres $0 \%$.

Die Lundsche Untersuchungsreihe hat uns gegenüber zweifellos den erheblichen Vorzug, daß sie bedeutend größer ist wie die unsrige.

Was unsere Serie an zahlenmäBigem Umfang entbehrt, ersetzt sie zweifellos durch die Sorgfalt der Materialauswahl. Gerade dadurch gewinnt aber unseres Erachtens die annähernde Übereinstimmung der beiderseitigen Resultate an Bedeutung, und es darf damit wohl angenommen werden, daß die tatsächliche durchschnittliche Erkrankungsziffer des Nervus VIII bei rezenter, Lues zwischen $20-25 \%$ gelegen ist.

Unsere Untersuchungen beschränkten sich bisher ausschließlich auf Anfangsbefunde vor Einleitung einer antiluetischen Kur, da es, uns zunächst einmal lediglich darauf ankam, an unserem eigenen Material nachzuprüfen, ob die von Kobrak angegebenen erstaunlich hohen Ziffern über die Häufigkeit luetischer symptomloser Akustikusschädigungen auch für unsere Fälle zutrafen oder nicht.

Die nächste Aufgabe wird für uns die sein, an Hand unseres Materials zu verfolgen, wie sich das weitere Schicksal dieser Luetiker uriter der Wirkung der von sachverständiger Seite durchgeführtén spezifischen Behandlung hinsichtlich ihrer Oktavusverhältnisse gestaltet.

Wir werden auch die Frage, ob zwischen Liquorbefund und Akustikusstörung eine innere Beziehung besteht, nicht außer Acht lassen und in dieser Hinsicht vor allem die Fälle mit noch negativem Wassermann beobachten.

Diese Untersuchurigen sind bereits im Gange, und wir behalten uns vor, über deren Ergebnisse später weiter zu berichten.

\section{Literatur.}

I. A. Alexander: „Die Erkrankungen des Nervus VIII im Frühstadium der Lues und die Beeinflußbarkeit des Nerven durch unsere kombinierte.Hg.Salvarsankur." Dermatolog. Wochenschrift 19I9, Bd. 69, Nr. 34 . 
Io WALTHER BRÜNING, Zur Frage der Schädigungen des Nervus VIII usw.

2. G. Alexander (Wien): "Die Syphilis des Gehörorgans". Handbuch der Geschlechtskrankheiten von Finger usw. I9I6, III. Bd, II. Teil.

3. Kobrak: "Untersuchungen des Nervus VIII bei rezenter Lues. Erregbarkeit vor und am Ende einer kombinierten Hg.-Salvarsankur". Passows Beiträge 1920, Bd. I4, H. I/2.

4. Robert Lund: ,Neurolabyrinthitis syphilitica. (Habilitationsschrift Kopenhagen 1920.) Ref. Internat. Centralblatt für Ohrenheilkunde und RhinoLaryngologie, Bd. I8, H. 9/10.

5. Meirowsky und Leven: ,Mißlungene Abortivbehandlung der sog. primären seronegativen Lues." Münchner med. Wochenschr. 1920, Nr. 36.

6. E. Nathan: „Neurorezidiv nach kombiniert behandelter seronegativer Primärsyphilis." Münchner med. Wochenschr. I92I, H. I6. 